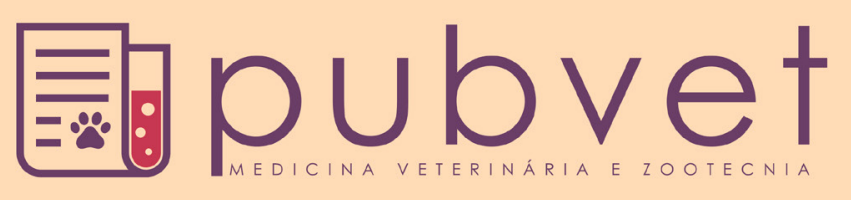

HTTP://DX.DOI.ORG/10.22256/PUBVET.V11N5.424-432

\title{
Cultivo de organismos aquáticos: uma proposta de desenvolvimento rural na Ilha de João Pilatos, Ananindeua - Pará - Brasil
}

\author{
Fabricio Nilo Lima da Silva ${ }^{1 *}$, Maria Sintia Monteiro da Costa ${ }^{2}$, Cínthia Silva Malcher ${ }^{2}$, \\ Luciano Ramos de Medeiros ${ }^{3}$, Antonia Rafaela Gonçalves Macedo ${ }^{4}$, Hugo Luiz Cordovil \\ de Freitas ${ }^{5}$, Raimundo Aderson Lobão de Souza ${ }^{6}$

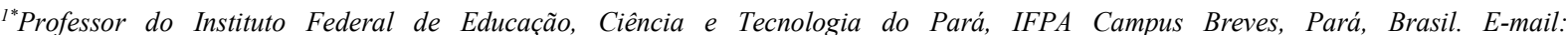 \\ fabricionilo@hotmail.com; \\ ${ }^{2}$ Mestra em Aquicultura e Recursos Aquáticos Tropicais pela Universidade Federal Rural da Amazônia, UFRA Campus Belém, Pará, Brasil. \\ E-mail: sintiamonteiro@hotmail.com; cinthiamalcher@yahoo.com.br. \\ ${ }^{3}$ Mestre em Desenvolvimento Rural e Gestão de Empreendimentos Agroalimentares pelo Instituto Federal de Educação, Ciência e Tecnologia \\ do Pará, IFPA Campus Castanhal, Pará,Brasil.E-mail: lucianomedeiros ses@hotmail.com; \\ ${ }^{4}$ Doutoranda do Programa de Pós-Graduação em Ciência Animal pela Universidade Federal do Pará, UFPA Campus Castanhal, Pará, \\ Brasil.E-mail: agmaquicultura@hotmail.com; \\ ${ }^{5}$ Professor do Instituto Federal de Educação, Ciência e Tecnologia do Pará, IFPA Campus Tucuruí, Pará, Brasil. E-mail: \\ freitashugo@hotmail.com; \\ ${ }^{6}$ Professor da Universidade Federal Rural da Amazônia, UFRA Campus Belém, Pará, Brasil.E-mail: adersonlobao@globo.com \\ *Autor para correspondência
}

RESUMO. O objetivo deste artigo foi conhecer o interesse dos agricultores familiares quanto à perspectiva da criação de organismos aquáticos em lotes agrícolas. A pesquisa foi conduzida na comunidade agroextrativista Nova Esperança, localizada na ilha João Pilatos no município de Ananindeua/PA. O percurso da pesquisa consistiu, em utilizar as ferramentas metodológicas do Diagnóstico Rural Participativo como as observações in loco, 30 entrevistas e caminhadas transversais. O diagnóstico mostrou que a maioria dos agricultores é do sexo masculino. Os mesmos compreendem que é importante implantar algum tipo de cultivo aquícola em seu lote, destacando a piscicultura em maior relevância. Há uma predominância da espécie exótica Tilápia-do-nilo, também foram identificadas espécies nativas como o Tambaqui e a Pirapitinga, e o híbrido "Tambacu" (fêmea de Tambaqui $\mathrm{x}$ macho do Pacu) como possíveis animais para o cultivo. Desse modo, na comunidade a atividade de piscicultura poderá ser desenvolvida pela agricultura de caráter familiar, tal atividade pode se configurar em uma possível alternativa para o fortalecimento das famílias e da economia local.

Palavras chave: Ilha, comunidade, agricultura familiar, alternativa de desenvolvimento, piscicultura

\section{Cultivation of aquatic organisms: a proposal for rural development in João Pilatos Island, Ananindeua - Pará - Brazil}

ABSTRACT. The objective of this article was to know the interest of the family farmers in the perspective of the creation of aquatic organisms in agricultural lots. The research was conducted in the agroextrativist Nova Esperança community, located on João Pilatos Island in the municipality of Ananindeua/PA. The methodological tools of Participatory Rural Appraisal as the on-site observations, 30 interviews and transect walks were used. The diagnosis revealed that the majority of farmers correspond to the male, to a lesser extent to the female gender. They declared find important to deploy some type of aquaculture farming in their lot, highlighting the most relevant in fish farming. There is a predominance 
of the exotic Oreochromis niloticus were also identified native species such as Colossoma macropomum and Piaractus brachypomus, and the hybrid "Piaractus mesopotamicus vs Colossoma macropomum" as possible animals for cultivation. Thus, community fish farming activity could be developed by the familiar character of agriculture, such activity can be configured in a possible alternative to strengthening families and the local economy.

Keywords: Island, community, agriculture, alternative, fish farming

\section{Producción de organismos acuáticos: una propuesta de desarrollo rural en la isla de João Pilatos, Anandeua - Pará - Brasil}

RESUMEN. El objetivo de este trabajo fue conocer los intereses de las familias agricultoras sobre la perspectiva de criar organismos acuáticos en lotes agrícolas. La investigación se realizó en la comunidad Agroextrativista Nova Esperanza, situada en la isla João Pilatos en el municipio de Ananindeua / PA. El desarrollo de la investigación consistió en el uso de herramientas metodológicas del Diagnóstico Rural Participativo como las observaciones sobre In loco, 30 entrevistas y caminadas transversales. El diagnóstico mostró que la mayoría de los agricultores son hombres. Ellos entienden que es importante implementar algún tipo de cultivo acuícola en su lote, destacando la piscicultura como el más relevante. Hay un predominio de la especie exótica Oreochromis niloticus, las especies nativas como Colossoma macropomum, la Piaractus brachypomus y el híbrido "Piaractus mesopotamicus vs Colossoma macropomum" también fueron identificadas como posible animales para el cultivo. Por lo tanto, en la comunidad la piscicultura podría ser desarrollada por la agricultura de carácter familiar, dicha actividad se puede configurar en una posible alternativa para el fortalecimiento de las familias y la economía local.

Palabras clave: Isla, comunidad, agricultura familiar, alternativa de desarrollo, cría de peces

\section{Introdução}

O desenvolvimento rural sustentável implica na desconstrução da associação que se faz do rural como o "atraso" e de imaginar que o desenvolvimento será um processo de urbanização do campo (Guimarães, 2011). Este mesmo autor aponta que as propostas contemporâneas de desenvolvimento rural sustentável estão pautadas no reconhecimento da diversidade, em políticas que estimulem a participação social e a dinamização da economia, com valorização dos recursos e atributos rurais, com respeito ao meio ambiente e aos valores culturais de cada comunidade.

No que diz respeito à diversificação da produção no meio rural, apresenta-se a aquicultura que vem se destacando no mundo inteiro, especialmente na Ásia e na América do Sul (ObaYoshioka et al., 2015). Sendo caracterizada como a produção de organismos com hábitats predominantemente aquáticos, em cativeiro, sob qualquer um de seus estágios de desenvolvimento (ovos, larvas, pós-larvas, juvenis ou adultos), tais como: peixes, algas, crustáceos, moluscos, quelônios e anfíbios, podendo ser realizadas em águas doces, estuarinas e salgadas
(Valenti, 2002, Macedo et al., 2016). Constituindo uma importante fonte de produção de proteína de origem animal com alto valor biológico (Lazzari et al., 2015).

A produção pesqueira mundial não apresentou crescimento significativo desde o ano de 2007, onde a produção foi de 90,8 milhões de toneladas até 2012 com produção de 91,3 , apresentando taxa de crescimento de $0,55 \%$ e em vista dessa estagnação, a aquicultura passou a ser apontada como a principal alternativa para incrementar a oferta de pescados, pois é importante destacar que neste mesmo período a produção cresceu em uma taxa de 33,46\%, passando de 49,9 milhões de toneladas de pescado em 2007 para 66,6 no ano de 2012 (FAO, 2015).

Segundo Araújo et al. (2015) esta prática tem se estabelecido como uma das mais promissoras atividades do agronegócio brasileiro, sendo a piscicultura seu mais importante ramo. De acordo com o Plano de Desenvolvimento da Aquicultura Brasileira - 2015/2020, o País é o $12^{\circ}$ maior produtor mundial em aquicultura, mas os 8.500 $\mathrm{km}$ de costa marítima e a maior reserva de água doce, entre outras características, que colocam em 
posição privilegiada para avançar muito nesse ranking (BRASIL, 2015).

Na região norte, atividade aquícola é menos desenvolvida em relação às demais regiões do país (Costa et al., 2014). Pesquisa de Rezende et al. (2008) destaca que a bacia Amazônica, é responsável por $20 \%$ da água doce do mundo, sobressaindo ainda os milhões de hectares de águas represadas em açudes e reservatórios e ainda a imensidão de seus mais de oito mil quilômetros de costa que possibilita uma enorme e variada atividade de cultivo de espécies marinhas. Favoráveis ao desenvolvimento desta aquicultura devido as suas características climáticas e hidrobiológicas (Araújo et al., 2015).

Por ser uma atividade de grande potencialidade na Amazônia Oriental mais precisamente realizada no Estado do Pará. Brabo (2014) considera a extensa rede hidrográfica, constituída por cursos d'água das bacias Amazônica, Araguaia-Tocantins e Atlântico Nordeste Ocidental, além de $562 \mathrm{~km}$ de litoral, que propiciam a prática de diversas modalidades de pesca e de aquicultura. No que diz respeito à criação de organismos aquáticos, Lee and Sarpedonti (2008) consideram que a atividade com destaque é a piscicultura.

Como na aquicultura brasileira, no Pará predomina a piscicultura continental, praticada em vários municípios, com modalidades de cultivos bem diversificados, desde a subsistência, até grandes produtores com a produção voltada para o mercado interestadual (De-Carvalho et al., 2013). Diante desta perspectiva, dentre as alternativas que pode proporcionar o incremento da atividade agrícola familiar, é a aquicultura em pequenos empreendimentos.

A agricultura familiar é uma forma sustentável de viver dos pequenos agricultores, ou seja, produzir os alimentos de maneira diversificada, cultivando várias espécies de plantas e animais numa mesma área, evitando a degradação do solo e do ambiente pelo uso da monocultura, utilizando a coletividade na comercialização e mão de obra como meio de produção (Neves et al., 2012). Portanto, a criação de organismos aquáticos é uma alternativa a ser adicionada como mais uma forma de produção na agricultura de base familiar, no sentido de garantir o sustento da família e o excedente para a comercialização.
De acordo com o pensamento de Castellani and Barrella (2005), a aquicultura está embasada em três pilares: a produção lucrativa, a preservação do meio ambiente e o desenvolvimento social, os três componentes são essenciais e indissociáveis para que se possa ter uma atividade perene. Portanto, torna-se necessário saber $\mathrm{o}$ interesse de agricultores e agricultoras familiares pela atividade de aquicultura a partir de uma compreensão ampla das realidades locais, e encontrar subsídios para gerar e transferir tecnologias compatíveis com esta realidade tornase necessário o conhecimento do perfil das mesmas, através de diagnóstico.

Assim, o cultivo de animais aquáticos pode contribuir para o desenvolvimento social e econômico da região onde se insere o município de Ananindeua/PA, localizada na Região Metropolitana de Belém, que possui ilhas e comunidades agrícolas, possibilitando o aproveitamento efetivo dos recursos naturais locais, principalmente os hídricos, e a criação de trabalho e renda proveniente da aquicultura.

Diante do exposto, este trabalho propõe conhecer o interesse dos agricultores e agricultoras familiares quanto à perspectiva da criação de organismos aquáticos na comunidade Agroextrativista Nova Esperança, Ananindeua/PA, pois essas informações são de grande importância para se transformar em uma atividade promissora para diminuir a sobrepesca de algumas espécies na Amazônia.

\section{Material e Métodos}

\section{Caracterização da área de estudo}

A pesquisa foi desenvolvida na comunidade Agroextrativista Nova Esperança, localizada na ilha João Pilatos no município de Ananindeua/PA. Este município pertence à região Metropolitana de Belém, possui aproximadamente $530 \mathrm{mil}$ habitantes e nove ilhas: João Pilatos, Viçosa, Sassunema, Mutá, Guajarina, São José da Sororoca, Sororoca, Arauari e Santa Rosa (Diniz, 2011). Ananindeua possui uma área aproximada de $176 \mathrm{Km}^{2}$, estando localizado entre as coordenadas geográficas $01^{\circ} 13^{\prime}$ e $01^{\circ} 27^{\prime}$ de Latitude Sul e $48^{\circ} 19^{\prime}$ e $48^{\circ} 26^{\prime}$ de Longitude Oeste de Greenwich. (Figura 1). O desenvolvimento deste trabalho ocorreu no período de agosto de 2013, totalizando 30 entrevistas nos pequenos empreendimentos agrícolas. 
MAPA DE LOCALIZAÇÃO
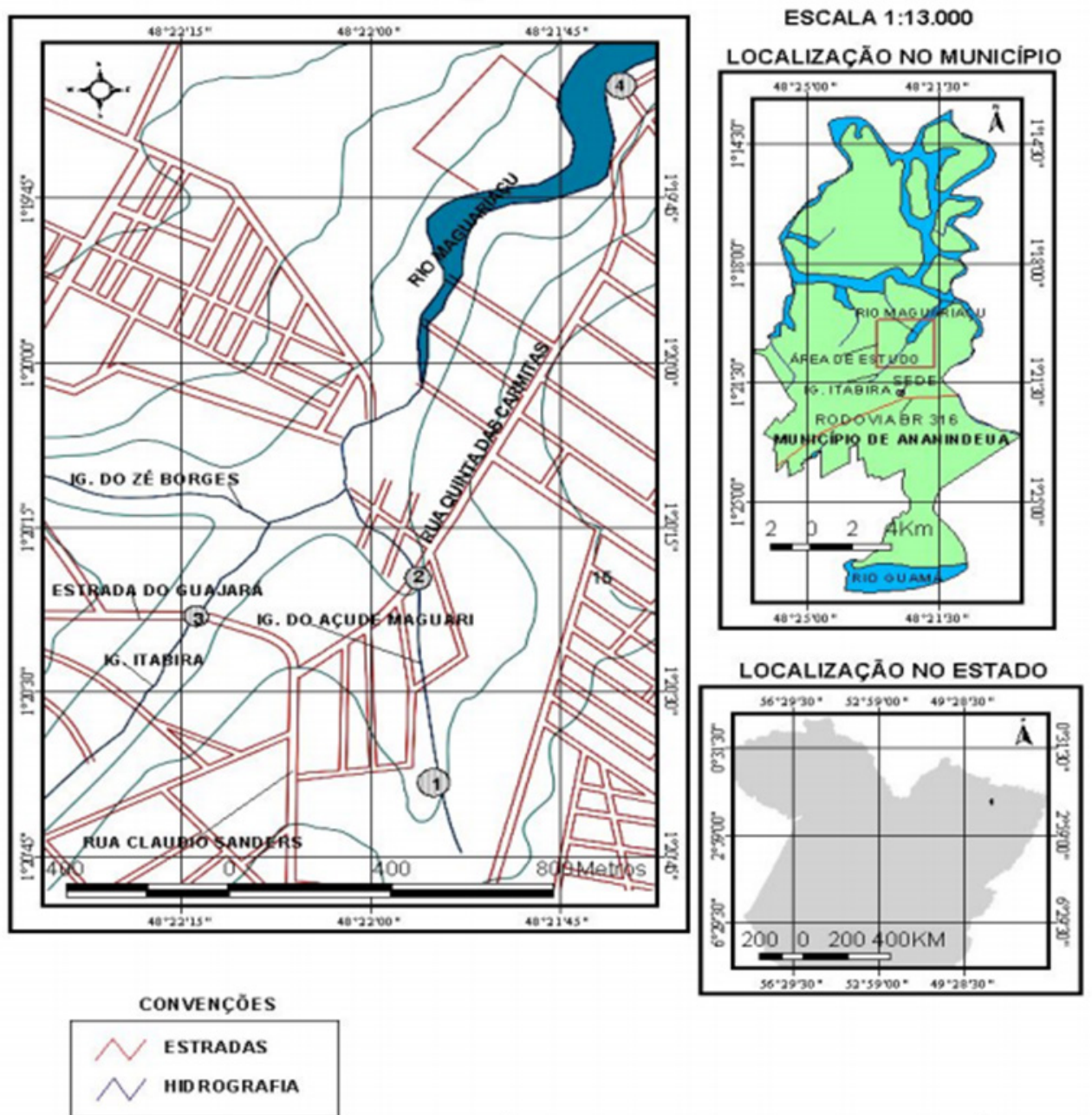

BASE : ISA / IBGE 2000

EXECUCCÃO: LABORATÓRIO DE ANÁLISE

DA INFORMACXXO GEOGRÁFICA - LAIG/UFPA

PROJETO CARTOG.: CARLOS TAMASAUSKAS DATA: $07 / 06 / 2006$

Figura 1. Mapa de localização do município de Ananindeua/PA. Fonte: adaptado de Pimentel et al., (2006).

\section{Obtenção e análise de dados}

O trabalho procedeu com uma abordagem qualitativa, com base de natureza exploratória, e pesquisa bibliográfica, além das visitas técnicas nos lotes agrícolas. Foi possível manter contato direto com os agricultores e do sistema de produção. Foram utilizadas as ferramentas metodológicas do Diagnóstico Rural Participativo (DRP) como as observações in loco, entrevistas e caminhadas transversais. Segundo Verdejo and Hidalgo (2003) é uma metodologia especificamente elaborada para intervenção, composta por uma conjugação de métodos e técnicas participativa, que possibilita a obtenção de informações qualitativas e quantitativas em curto espaço de tempo. Constitui um instrumento metodológico de identificação de problemas, suas causas e possíveis soluções a partir da interação dialógica entre os atores sociais.

No que diz respeito à entrevista semiestruturada, a atenção tem sido dada principalmente à formulação de perguntas básicas para o tema a ser investigado (Manzini, 2003, Triviños, 2015). O formulário da entrevista contou com perguntas socioeconômicas, com questões relativas a nome, sexo, idade, naturalidade, escolaridade, estado civil, atividade principal e secundária de renda do agricultor, caracterização da propriedade e levantamentos de dados no que se refere à perspectiva da atividade de aquicultura familiar dentro da comunidade, e dentre outros dados relevantes necessários para traçar o perfil na ilha.

As informações relativas aos perfis socioeconômicos e da comercialização foram 
agrupadas e analisadas de forma qualitativa e quantitativa.

\section{Resultados e Discussão}

Foi evidenciado que a maioria $(70 \%)$ dos produtores entrevistados, correspondeu ao sexo masculino e a menor parte $(30 \%)$ ao feminino, sendo potenciais para desenvolver alguma atividade aquícola. Destes, (60\%) dos agricultores(as) são casados, (20\%) encontram-se divorciados e (20\%) não informaram. Resultados de Silva et al. (2010) trabalhando com a piscicultura familiar do tambaqui na região do baixo São Francisco concluíram que em sua maioria evidenciaram homens gerenciando a atividade, no entanto mulheres também foram encontradas desempenhando tal função, pesquisa esta, não foi diferente a do presente estudo.

Para Maneschy (2000), as atividades desenvolvidas pelas mulheres, no que diz respeito dos recursos pesqueiros, apresenta uma tendência a ser multidirecionadas, enquanto as dos homens normalmente são centradas em uma ou duas atividades. Com relação ao grau de escolaridade, foi verificado que mais da metade $(67 \%)$ dos futuros aquicultores possui o ensino fundamental incompleto e apenas uma pequena parte $(20 \%)$ com o ensino fundamental completo (Gráfico1).

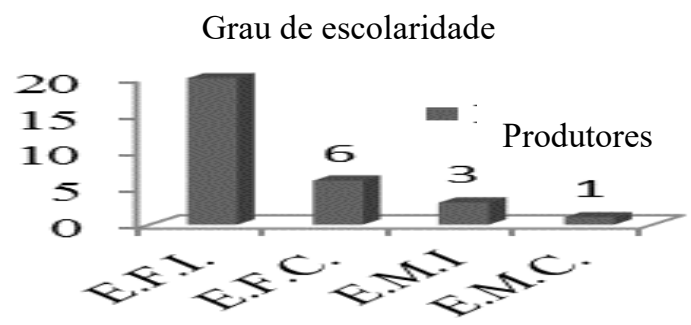

Gráfico 1: Grau de escolaridade (E.F.I. - Ensino Fundamental Incompleto; E.F.C. - Ensino Fundamental Completo; E.M.I. - Ensino Médio Incompleto e E.M.C. Ensino Médio Completo) dos produtores na comunidade Agroextrativista Nova Esperança/PA.

Silva et al. (2011) ao realizarem levantamento sobre a piscicultura na mesorregião Sudeste do Estado do Pará, a pesquisa apontou que os piscicultores não possuem instrução formal ou possui ensino fundamental incompleto, alegando que não tiveram oportunidade de estudar devido desde cedo auxiliam no sustento da família. Pesquisa conduzida por Sarah et al. (2013) trabalhando com aspectos da atividade de piscicultura praticada por produtores rurais no município de Cruzeiro do Sul (Acre), o grau de escolaridade da maioria dos produtores é o ensino fundamental incompleto, colaborando com a do presente estudo.

As idades variaram entre 27 a 76 anos, com a maioria dos agricultores(as) (70\%) com idades entre 49 e 55 anos, demonstrando que possivelmente a atividade aquícola será proporcionalmente menos explorada por pessoas mais jovens em atividades relacionadas ao campo. Macedo et al. (2016) traçaram o perfil da ostreicultura na comunidade de Santo Antônio do Urindeua, Salinópolis, nordeste do Pará, segundo a fala de um jovem da comunidade, o motivo pelo qual eles não estão envolvidos na atividade está relacionado ao receio em exercer a profissão, pois eles se sentem menosprezados perante aos jovens da cidade.

A renda familiar média dos produtores é inferior a um salário e meio. Quanto ao tamanho das propriedades, elas são pequenas propriedades com no máximo 2 ha, tamanho este considerado interessante para desenvolver alguma atividade aquícola de base familiar. Souza et al. (2015) trabalhando com a piscicultura no Marajó/PA, em média foi de dois salários por família e 6,2 ha de áreas sendo utilizada na agricultura e pecuária, superior aos encontrados na presente pesquisa.

Do total de entrevistados $(60 \%)$ apresenta a agricultura como a principal atividade de renda com o cultivo da horticultura, fruticultura, suinocultura, avicultura, além do cultivo de plantas ornamentais e medicinais, o restante provém da atividade de pesca e agricultura com $(27 \%)$ e somente $(13 \%)$ da atividade de pesca contribui tanto para venda quanto para subsistência na localidade (Gráfico 2).

Resultados obtidos neste estudo ratificaram aos encontrados por Corrêa et al. (2008) caracterizando a piscicultura no Vale do Ribeira/SP, que nem sempre a aquicultura é a única atividade realizada na propriedade. Assim, ao se pensar na criação de organismos aquáticos como uma opção nos empreendimentos locais, existe a necessidade de motivar formas de organização como o associativismo ou cooperativismo, para torná-los viáveis do ponto de vista econômico. Neta perspectivas, os agricultores e agricultoras da comunidade fundaram uma associação denominada "Pequenos Produtores Rurais da Comunidade Nova esperança", deste modo, esta organização possivelmente tornará importante para poder começar as atividades de forma coletiva. 


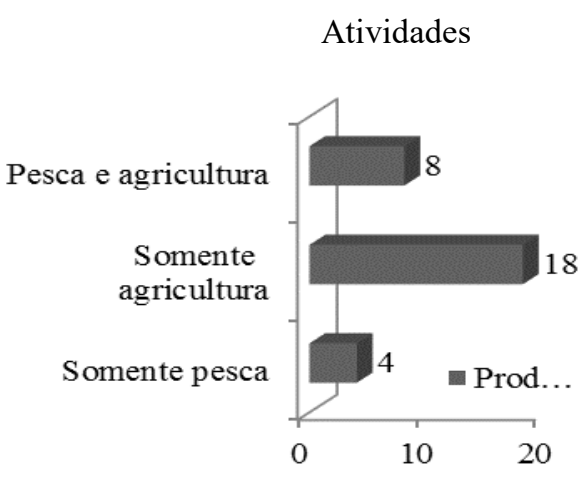

Gráfico 2: Atividades desenvolvidas na comunidade Agroextrativista Nova Esperança/PA.

Portanto, a associação de futuros aquicultores visando à rentabilidade na cadeia de produção torna-se importante na localidade. Sarah et al. (2013) destacam que uma associação ou cooperativa de produtores reduz custos logísticos, o que, consequentemente, aumenta o lucro da venda. Essa experiência foram evidenciadas na pesquisa de Brabo (2014) trabalhando com a caracterização do cultivo de pirarucu no município de Conceição do Araguaia/PA,a maioria dos piscicultores mostraram interesse em participar de uma associação ou cooperativa.

Do total de entrevistados, todos são nascidos no estado do Pará, prevalece pessoas nascidas na cidade de Ananindeua com (37\%), Belém (23\%), Bragança $(10 \%)$, Cametá (13\%), Capitão Poço (7\%), Ourém (7\%) e Oeiras do Pará (3\%). Todos tem interesse em implantar algum tipo de cultivo em seu lote familiar (Gráfico 3), (60\%) das respostas diz respeito à piscicultura, (27\%) carcinicultura, (10\%), quelonicultura e (3\%) com ostreicultura, sendo que os demais cultivos não foram mencionados.

A aquicultura pode ser realizada em estruturas de pequena escala (familiares), com finalidades comerciais e de reserva de alimentos (Schirmer and Cardoso, 2010). A aquicultura paraense abrange às atividades de piscicultura continental, carcinicultura marinha e ostreicultura, apesar de já ter contado com empreendimentos comerciais de carcinicultura de água doce, com o camarãogigante-da-Malásia Macrobrachium rosenbergii, de ranicultura, com a rã-touro Lithobates catesbeianus, e de quelonicultura, com a tartaruga-da- Amazônia Podocnemis expansa (Brabo, 2014).
Interesse pela atividade

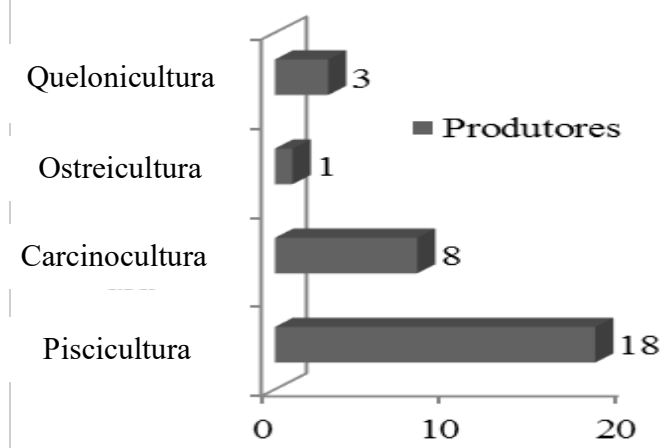

Gráfico 3. Perspectivas de culturas de preferência para instalar na comunidade Agroextrativista Nova Esperança/PA.

No que diz respeito à opinião dos agricultores em relação à importância da atividade aquícola nos lotes agrícolas, todos concordam que a mesma é necessária, sendo a piscicultura a atividade de maior interesse. Esta atividade foi evidenciada também por Piedras and Bager (2007) quando caracterizaram a aquicultura Rio Grande do Sul; no Nordeste Paraense por O'de Almeida-Júnior and Lobão (2013) e a mais recente pesquisa de Lima and Shinozaki-Mendes (2015) estudando o perfil abiótico e viabilidade à aquicultura no açude do Saco, sertão do Pajeú (Pernambuco). Estes resultados corroboram com a afirmação de Ostrensky et al. (2008) que consideram a piscicultura no Brasil como a mais desenvolvida por pequenos produtores rurais.

Quando perguntados sobre a importância da piscicultura na comunidade Nova Esperança. Os mesmos foram enfáticos em dizer que adotaria em seus lotes, tais como: "a criação de peixes é bom nos quintais" (agricultor, 76 anos); "sabendo cultivar direito os peixes vai pra frente, pois temos a grande riqueza que é a água" (agricultor, 44 anos); "gostaria de cultivar os peixes para o sustendo da minha família e também para vender" (agricultor, 49 anos); "eu gosto muito de peixe e minha familia também, e tiver peixe pra criar vai ser muito bom" (agricultora, 55 anos).

Nesse sentido, a aquicultura é entendida como uma atividade agrícola (Garutti, 2003) e a piscicultura se destaca como uma alternativa a ser experimentada na agricultura familiar, pela grande produtividade por área e também por ter como produto final um alimento proteico de alto valor nutricional (Baccarin et al., 2009). As opiniões dos agricultores familiares demonstram que a atividade aquícola é interessante na comunidade, tendo conhecimento sobre a real situação dessa atividade no Estado. Quando perguntados sobre a 
experiência de algum tipo de cultivo aquícola, $(67 \%)$ dos entrevistados já tiveram contato com a atividade, sendo o maior destaque a piscicultura e (33\%) não souberam informar.

A pesquisa mostrou que há uma predominância de cultivar nos lotes agrícolas a espécie exótica tilápia-do-nilo (Oreochromis niloticus Linneaus, 1758 ) com (46\%) citada pelos entrevistados em seguida as espécies popularmente chamadas de "redondos", dentre eles: o tambaqui (Colossoma macropomum Cuvier, 1818) com (27\%) e a pirapitinga (Piaractus brachypomus Cuvier, 1818) com (17\%), e de seu híbrido "tambacu" (ㅇ de tambaqui x ${ }^{\Uparrow}$ do pacu) com (10\%) (Gráfico 4). Além dessas espécies mencionadas, Brabo (2014) destaca que no Pará pode encontrar cultivos de tambatinga $C$. macropomum $\odot$ × $P$. brachypomus $\widehat{\jmath}$, pirarucu, surubim Pseudoplatystoma spp., matrinxã Brycon amazonicus, piau Leporinus spp. e o curimatã Prochilodus spp. que podem ser implementados na comunidade local.

\section{Possíveis espécies}

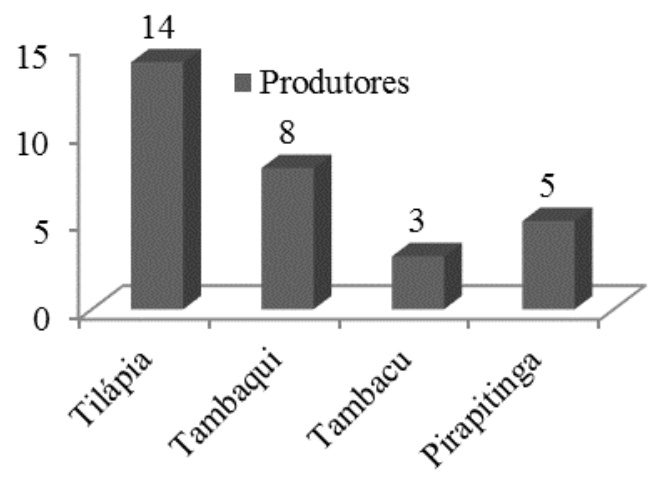

Gráfico 4: Perspectivas de espécies de peixes de preferência para cultivar na comunidade Agroextrativista Nova Esperança/PA.

As perspectivas dos cultivos de organismos aquáticos na comunidade Nova Esperança apresenta potencialidade de crescimento do ponto de vista das condições ambientais, pois poderá contribuir no sentido de alavancar com o agronegócio no Estado, porém a aquicultura paraense apresenta grandes gargalos, no caso da quelonicultura Martins et al. (2003) trabalhando com cultivo de tartaruga-da-Amazônia, como uma alternativa de produção para a aquicultura no estado do Pará, afirmam que entre os maiores entraves relatados pelo produtor, destacam-se: alto custo para aquisição de insumos, principalmente para a alimentação, tendo em vista que não existe uma ração adequada que atenda as exigências nutricionais da espécie; os animais devem ser comercializados vivos, não podendo ser processados devido à falta de abatedouros específicos; o produto ainda apresenta baixa aceitação no mercado; e carência de subsídios e assistência técnica.

No que diz respeito à atividade de ostreicultura que é recente no Pará, sendo praticada a pouco mais de uma década. Atualmente, esta vem se tornando uma das principais alternativas de geração de renda no Estado. Macedo et al. (2016) enfatiza que apesar desse avanço, a ostreicultura no Pará ainda se apresenta como atividade secundária para pescadores artesanais e agricultores familiares, pois não são capazes de desenvolver a economia local exclusivamente do cultivo de ostras. A piscicultura continental é o ramo da aquicultura mais desenvolvido no Pará (Brabo, 2014, Souza et al., 2015) e compreendem que pode ser de forma extensiva e de subsistência, utilizando principalmente a mão de obra familiar um dos problemas encontrados destaca-se o roubo, assistência técnica, legislação e ração.

Neste sentido, percebe-se o interesse dos agricultores familiares em desenvolver atividades de aquicultura na comunidade de Nova Esperança. Para alavancar essas atividades se faz necessária a junção do poder público, no sentido de oferecer condições logísticas para o escoamento da produção; o poder privado investindo em planta de beneficiamento e formas a traçar estratégias que viabilizem o acesso ao crédito, e o setor acadêmico realizando pesquisas bem como assistência e capacitação aos produtores locais.

\section{Conclusão}

$\mathrm{Na}$ percepção dos agricultores familiares a criação de organismos aquáticos é de grande importância na comunidade de Nova Esperança, destacando em maior relevância a introdução da piscicultura nos lotes agrícolas. Há uma tendência de cultivar a tilápia-do-nilo, tambaqui e a pirapitinga e de seu híbrido "tambacu" (fêmea de tambaqui $\mathrm{x}$ macho do pacu).

Foi diagnosticado que esta atividade apresenta um grande potencial para ser implantada apenas de caráter de subsistência, no sentido de garantir a inserção de proteína de alta qualidade (pescado) na mesa da família e o excedente como forma comercial.

Portanto, é necessária a adoção de ações governamentais no sentido de desenvolver estratégias viáveis como: o acesso a capacitação técnica e a disponibilidade de crédito aos futuros 
aquicultores. Desse modo, a piscicultura em pequenos empreendimentos na comunidade Nova Esperança, pode ser uma possível alternativa para o fortalecimento da economia local.

\section{Agradecimentos}

A todos os agricultores na comunidade Nova Esperança do Município de Ananindeua/PA pela contribuição na pesquisa realizada.

\section{Referências bibliográficas}

Araújo, J. G., Santos, M. A. S., Rebello, F. K., Oliveira, C. M. \& Costa, A. D. 2015. Crédito rural para aquicultura: uma análise do Fundo Constitucional de Financiamento do Norte no estado do Pará. Electronic Journal of Management, Education and Environmental Technology, 19, 553-562.

Baccarin, A. E., Leonardo, A. F. G., Tachibana, L. \& Correia, C. F. 2009. Piscicultura em comunidade remanescente de quilombo: um estudo de caso. Informações Econômicas, São Paulo, 39, 42-47.

Brabo, M. F. 2014. Piscicultura no Estado do Pará: situação atual e perspectivas. Acta of Fisheries and Aquatic Resources, 2, 1-7.

Castellani, D. \& Barrella, W. 2005. Caracterização da piscicultura na região do Vale do Ribeira-SP. Ciência e Agrotecnologia, 29, 168-176.

Corrêa, C. F., Scorvo Filho, J., Tachibana, L. \& Leonardo, A. F. G. 2008. Caracterização e situação atual da cadeia de produção da piscicultura no Vale do Ribeira. Informações Econômicas, 38, 30-36.

Costa, L. C. O., Brito, T. P., Macedo, A. R., Oliveira Sampaio, L. d. S., Silva, F. N. \& Silva, M. C. A. 2014. A perspectiva de alunos do curso técnico em agropecuária em relação à aquicultura. Ambiência, 10, 707-721.

De-Carvalho, H. R. L., Souza, R. A. L. \& Cintra, I. H. A. 2013. A aquicultura na microrregião do Guamá, Estado do Pará, Amazônia Oriental, Brasil. Ciências Agrárias, 56, 1-6.

Diniz, F. P. S. 2011. Territótio e desenvolvimaneot sustentável no assentamento agro-extrativista Nova Esperança-Ilha João Pilatos/Ananienua-PA. Anais das Jornadas de
Pós-Graduação da Faculdade Integrada Brasil Amazônia-FIBRA.

FAO. 2015. Statistical Yearbook. Food and Agriculture Organization of the United Nations, Rome, Italy.

Garutti, V. 2003. Piscicultura ecológica. UNESP, São Paulo.

Guimarães, M. D. A. 2011. Desenvolvimento de territórios rurais no Brasil: uma estratégia inovadora para o desenvolvimento rural sustentável. In: Nascimento, P. P., H., S., Martins, M. A. G., Balsadi, O. V. \& Silva Júnior, C. D. (eds.) Inovações em desenvolvimento territorial: novos desafios para a Embrapa. Brasília, DF: Embrapa Informação Tecnológica. EMBRAPA, Brasília, Brasil.

Lazzari, R., Uczay, J., Rodrigues, R. B., Pianesso, D., Adorian, T. J. \& Mombach, P. I. 2015. Utilização de resíduos de frutas em dietas para piava. Boletim do Instituto de Pesca, 41, 227237.

Lee, J. R. \& Sarpedonti, V. 2008. Diagnóstico, tendência, potencial e políticas públicas para o desenvolvimento da aquicultura. Diagnóstico da pesca e da aquicultura no Estado do Pará, 1, 1-109.

Lima, J. R. C. \& Shinozaki-Mendes, R. A. 2015. Perfil abiótico e viabilidade à aquicultura no açude do Saco, sertão do Pajeú, Pernambuco. Brazilian Journal of Agricultural Sciences, 10, 153-158.

Macedo, A. R. G., Silva, F., Ribeiro, S. d. C., Torres, M. F., Silva, F. N. L. d. \& de Medeiros, L. R. 2016. Perfil da ostreicultura na comunidade de Santo Antão Nio do Urindeua, Salinópolis, Nordeste Do Parã/Brasil. Observatorio de la Economía Latinoamericana, 217, 1-10.

Maneschy, M. C. 2000. Da casa ao mar: papéis das mulheres na construção da pesca responsável. Proposta, 84, 82-91.

Manzini, E. J. 2003. Considerações sobre a elaboração de roteiro para entrevista semiestruturada. Colóquios sobre pesquisa em educação especial, 11-25.

Martins, R. T., Barreto, L. \& Pereira-Martins, S. R. 2003. Onservação de aniomalias congênitas em tartarugas de água doce Trachemys adiutrix (Vanzolini, 1995) em ambientes naturais. 
Boletim do Laboratório de Hidrobiologia, 16, 75-77.

Neves, M. F., Mussury, L. L. A. \& Mussury, R. M. 2012. Análise do processo de diversificação e agregação agroecológico do assentamento Itamarati-MS. Comunicação \& Mercado, 1, 85.

O’de Almeida-Júnior, C. R. M. \& Lobão, R. A. 2013. Aquicultura no Nordeste paraense, Amazônia Oriental, Brasil. Boletim Técnico Científico do Cepnor, 13, 33-42.

Oba-Yoshioka, E. T., de Almeida, R. S., Gemaque, S. R. F., Brasiliense, A. R. P., Souza Silva, R. \& Marinho, R. d. G. B. 2015. Substituição parcial da ração comercial por soja e milho cozidos e sua influência sobre o cultivo de híbridos tambatingas. Biota Amazônia, 5, 61-67.

Ostrensky, A., Borghetti, J. R. \& Soto, D. 2008. Aqüicultura no Brasil: o desafio é crescer. Secretaria Especial de Aqüicultura e Pesca: FAO.

Piedras, S. R. \& Bager, A. 2007. Caracterização da aqüicultura desenvolvida na Região Sul do Rio Grande do Sul. Current Agricultural Science and Technology, 13, 403-407.

Rezende, F. J. W., Silva, J. B., Mello, C. F., Souza, R. A. L., Souza, A. S. \& Kloster, A. C. 2008. Perfil da aquicultura no estado do Acre. Amazônia: Ciência \& Desenvolvimento, 4, 167-180.

Sarah, M. G. M., Santos, M. I. S., Souza, L. P. \& Santiago, A. C. C. 2013. Aspectos da atividade de piscicultura praticada por produtores rurais no município de Cruzeiro do Sul - Acre. Enciclopédia Biosfera, 9, 568.

Schirmer, G. J. \& Cardoso, E. S. 2010. A piscicultura na dinâmica socioeconômica do município de Agudo-RS. Boletim Gaúcho de Geografia, 36, 23-28.
Silva, A. M. C. B., Souza, R. A. L., Melo, Y. P. C., Zacardi, D. M., Paiva, R. S. \& Nakayama, L. 2010. Diagnóstico da piscicultura na mesorregião Sudeste do Estado do Pará. Boletim Técnico Científico do Cepnor, 10, 5565.

Silva, N. J. R., Lopes, M. C., Fernandes, J. B. K. \& Henriques, M. B. 2011. Caracterização dos sistemas de criação e da cadeia produtiva do lambari no Estado de São Paulo, Brasil. Informações Econômicas. São Paulo, 41, 1728.

Souza, R. A. L., Souza, A. S. L., Silva, F. N. L., Souza, F. B., Aranha, T. V. \& Lopes, A. S. 2015. A Piscicultura no Marajó, Pará, Amazônia Oriental, Brasil. Boletim Técnico Científico do Cepnor, 15, 23-29.

Triviños, A. N. S. 2015. Introdução à pesquisa em ciências sociais: a pesquisa qualitativa em educação. O positivismo; a fenomenologia; o marxismo. Atlas, São Paulo.

Valenti, W. C. 2002. Aquicultura sustentável. In: Zootécnicos, A. P. d. E. (ed.) Congresso de Zootecnia. Portugal.

Verdejo, M. E. \& Hidalgo, E. J. 2003. Diagnóstico rural participativo: Una guía práctica. Centro Cultural Poveda, Proyecto Comunicación y Didáctica, Brasília.

\section{Article History:}

Received 6 February 2017

Accepted 7 March 2017

Available on line 28 April 2017

License information: This is an open-access article distributed under the terms of the Creative Commons Attribution License 4.0, which permits unrestricted use, distribution, and reproduction in any medium, provided the original work is properly cited. 